\title{
Informer le maximum de personnes responsables
}

Stéphane Reymond

Correspondance:

Dr Stéphane Reymond

Rue du Petit-Bois 11

CH-2316 Les Ponts-de-Martel

stephane.reymond(at)gmail.com
Le 21 mars 2011, j'informais Monsieur le Conseiller fédéral Didier Burkhalter de la disparition future des cabinets individuels de campagne, ceci pour raison économique.

Plusieurs années seront encore nécessaires pour que des cabinets de groupe les remplacent, pour autant que le tarif soit aussi adapté.

Pour un revenu AVS de 200000 franc par an en travaillant 42 heures la semaine, sans laboratoire, le point TARMED devrait se situer à 1.40. Actuellement, il reste $22 \%$ du chiffre d'affaires après déduction de l'AVS et des cotisations pour la retraite. La lettre avec le graphique des pourcentages est à disposition sur le site www.primary-care.ch.

Suite à la large diffusion de cette lettre (autorités fédérales, cantonales, au monde politique suisse, aux médias), de nombreuses réactions me sont parvenues.

Dans sa réponse, $\mathrm{M}$. Didier Burkhalter rappelle que «le Conseil fédéral est chargé de vérifier que les conventions tarifaires s'étendant à toute la Suisse sont conformes à la loi et à l'équité et qu'elles satisfont au principe d'économie (art. 46, al. 4, LAMal). Les partenaires tarifaires sont en train de la réviser pour que la médecine de base soit mieux valorisée. Je suis ces travaux de près et avec un grand intérêt. En fait, j'ai invité les partenaires tarifaires à m'informer sur les travaux en cours et sur la date butoir tout en étant disposé à m'engager si nécessaire en faveur de la révision.»

Il ajoute que le «Managed Care a notamment pour objectif de revaloriser le rôle du médecin de premier recours».

M. Pierre-Yves Maillard, Président de la CDS, «partage mon inquiétude et va la relayer encore davantage».

M. Jacques de Haller, Président de la FMH, «espère une réponse constructive».

Les parlementaires contactés, quelque soit leur appartenance politique, reconnaissent le problème et vont soutenir les mesures utiles à l'amélioration de la situation. Parmi les réponses des présidents des partis suisses, M. Fulvio Pelli «partage que, dans un souci d'efficience, les cabinets de groupe doivent se développer. Cela ne peut se faire sans égard aux personnes qui assument encore un cabinet individuel.» En Suisse encore $2 / 3$ des médecins de famille travaillent de manière individuelle.
Mme Gisèle Ory, Conseillère d'Etat NE, rappelle que «le Conseil d'Etat a jusqu'ici, dans le cadre de la procédure de fixation de la valeur du point TARMED, veillé à ce que les médecins neuchâtelois puissent maintenir des conditions financières stables. Notre canton participe au Programme d'assistanat en cabinet.» Elle ajoute que «la législation ne nous donne pas de compétence en matière de planification de la médecine ambulatoire... et de subventionnement...»

Les autorités communales contactées ont été très réceptives et répondent qu'elles soutiendront les installations de médecins de famille, le cas échéant.

\section{«Depuis cet été, une évolution positive se dessine.»}

Plusieurs collègues, également de Suisse alémanique, m'ont répondu que les pourcentages correspondaient grosso modo aux leurs et qu'ils devaient beaucoup travailler pour vivre correctement. Tous soutiennent la démarche.

Depuis cet été, une évolution positive se dessine. Dans son communiqué du 16.9.2011, le Conseil fédéral vient de décider (initiative populaire «Oui à la médecine de famille»: transmission du message avec un contre-projet, al. 5) «de prolonger pour 2012 le supplément de transition pour les laboratoires en cabinet» et de soutenir «une initiative parlementaire l'habilitant à procéder à des adaptations de la structure tarifaire, si celle-ci s'avère inappropriée et si les partenaires ne peuvent s'entendre sur une révision».

Pour agir rapidement, il serait opportun de créer une nouvelle position TARMED, utilisable de suite: «prise en charge par le médecin de famille» de l'ordre de 20 francs, valable aussi pour les pédiatres, qui serait utilisée à chaque consultation et s'ajouterait au temps calculé actuellement.

Informer le maximum de personnes responsables au niveau des autorités politiques me paraît important. 